\title{
Possible Delayed Recurrence of Sporadic Spinal Hemangioblastoma after Complete Surgical Resection: A Case Report
}

\author{
Bongmin Jung ${ }^{1}$, Soo Yeon Kim², Woo Jin $\mathrm{Choe}^{1}$, So Dug Lim³ \\ ${ }^{1}$ Department of Neurosurgery, Konkuk University Medical Center, Konkuk University School of Medicine, Seoul, Republic of Korea \\ ${ }^{2}$ Department of Neurosurgery, Kangdong Sacred Heart Hospital, Hallym University College of Medicine, Seoul, Republic of Korea \\ ${ }^{3}$ Department of Pathology, Konkuk University Medical Center, Konkuk University School of Medicine, Seoul, Republic of Korea
}

Corresponding author: Woo Jin Choe

Department of Neurosurgery, Konkuk University Medical Center, Konkuk University School of Medicine, 120-1

Neungdong-ro, Gwangjin-gu, Seoul 05030, Republic of Korea

Tel: +82-2-2030-7625

Fax: +82-2-2030-7359

E-mail: s1nsman@chol.com

Received: August 26, 2021

Revised: September 23, 2021

Accepted: September 24, 2021

\begin{abstract}
Spinal cord hemangioblastoma (HBL) is a highly vascularized tumor that occurs sporadically or in relation to von Hippel-Lindau disease. Sporadic-type HBLs in the spinal cord usually present as single lesions that are potentially curable by complete surgical resection, with a low rate of recurrence. We report a case of a totally excised sporadic-type spinal cord $\mathrm{HBL}$ with multiple recurrences observed 10 years after surgery. Long-term follow-up may be necessary for spinal cord $\mathrm{HBL}$, even in cases of complete resection.
\end{abstract}

Key Words: Hemangioblastoma; Recurrence; Spinal cord

\section{INTRODUCTION}

Spinal cord hemangioblastoma (HBL) is a benign, slow-growing tumor that consists of stromal cells and small blood vessels ${ }^{11}$. HBL accounts for $2 \%$ to $15 \%$ of primary tumors in the spinal cord, and it arises either sporadically or in relation to von Hippel-Lindau disease $(\mathrm{MHL})^{7,14,17}$. $\mathrm{VHL}$ is a familial neoplastic condition caused by germline mutations of the tumor suppressor gene $\mathrm{VHL}(3 \mathrm{p} 25)^{8,16}$ in most cases. $\mathrm{VHL}$ could occur via de novo mutation of the VHL gene in approximately $20 \%$ of the cases. In VHL patients, tumors can occur in the kidneys, adrenal glands, pancreas, reproductive organs, retina and central nervous system (CNS) including spinal cord ${ }^{16)}$. Approximately two- thirds of spinal HBLs are sporadic, and $80 \%$ of HBLs are solitary. $\mathrm{VHL}$ syndrome is suspected in the presence of multiple lesions.

Surgical treatment should be considered for patients with neurologic symptoms ${ }^{10}$. Complete surgical resection is usually curative, sometimes with the aid of preoperative embolization for large tumors". Recurrence has been reported in up to $25 \%$ of patients with CNS $\mathrm{HBLs}$, and recurrence of sporadic HBL after gross total resection (GTR) is rare. Recurrences of sporadic HBLs have been associated with prior incomplete resection ${ }^{13)}$. In this study, we describe a case of multiple recurrent spinal HBLs 10 years after GTR in a patient, despite the absence of evidence of residual tumor 1 year after the operation.

\section{CASE REPORT}

A 54-year-old male patient visited the outpatient department complaining of bilateral buttock pain that had been present for
5 months. Magnetic resonance (MR) images revealed a 2.6-cm hypervascular intrathecal tumor at the L3 level, with tortuous venous engorgement from $\mathrm{TlO}$ to the sacrum. A spot-like lesion within the venous engorgement was observed above the tumor at L2-3 level. The tumor characteristics appeared consistent with spinal cord $\mathrm{HBL}$ on the basis of findings from imaging studies (Fig. 1). A spot-like lesion was noted and it was thought to be either cross section of venous engorgement or a daughter nodule.

Physical and radiological examinations, including fundus exam, ultrasonography of the abdominopelvic organ, brain and whole spine MR were proceeded related to VHL syndrome. The results of the tests showed no abnormality. VHL is diagnosed if the patient has a family history of $\mathrm{VHL}$ and presence of one $\mathrm{VHL}$ associated tumor. If the patient has no family history of $\mathrm{VHL}$, multiple $\mathrm{VHL}$
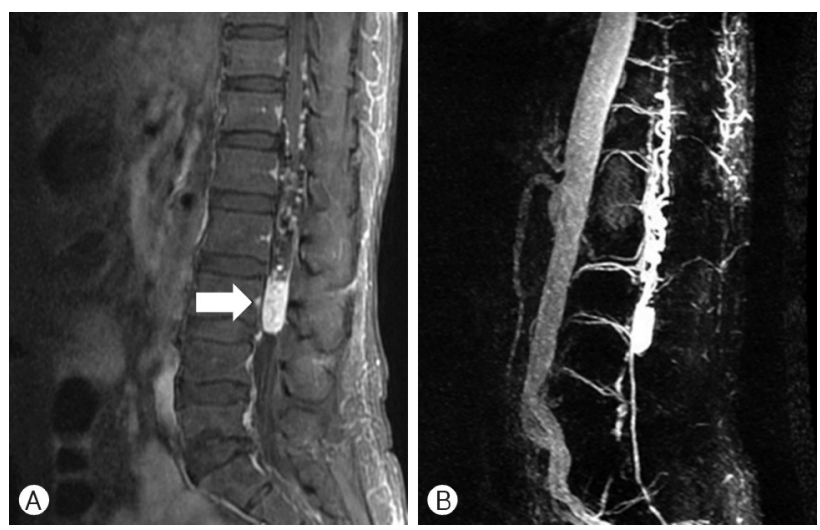

Fig. 1. (A) Magnetic resonance image showed a homogenous 2.6 $\mathrm{cm}$ sized well enhancing mass at $\mathrm{L} 3$ level and (B) tortuous vascular structure from $\mathrm{T} 10$ to sacrum is observed. 

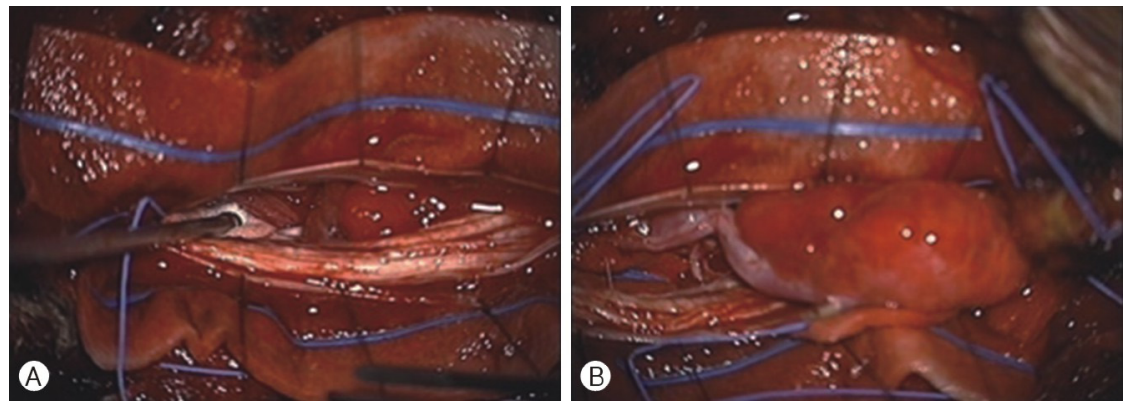

Fig. 2. Microscopic view of the spinal hemangioblastoma. The tumor was highly vascularized and had solid multiloculated pattern. The tumor adhered to the filum terminale, showed no infiltration to surrounding tissues. The tumor was completely removed by en bloc pattern.
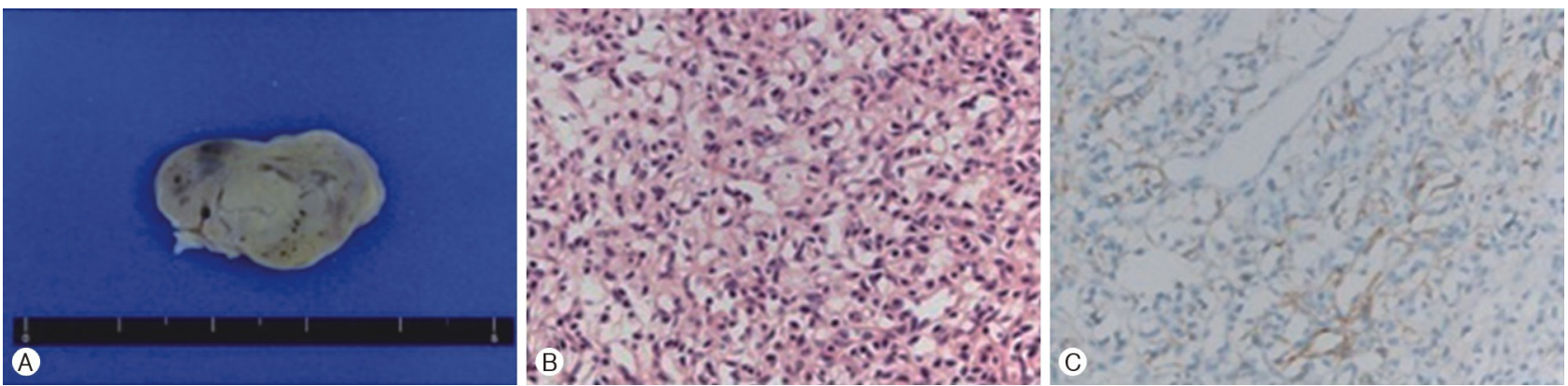

Fig. 3. Tumor tissue obtained from the operation. Microscopic study of the tumor tissue showed stromal cells with clear foamy cytoplasm ( $\times 400$ magnification, middle), and CD56 stained stromal cells ( $\times 400$ magnification, right). The pathological study was consistent with a hemangioblastoma.
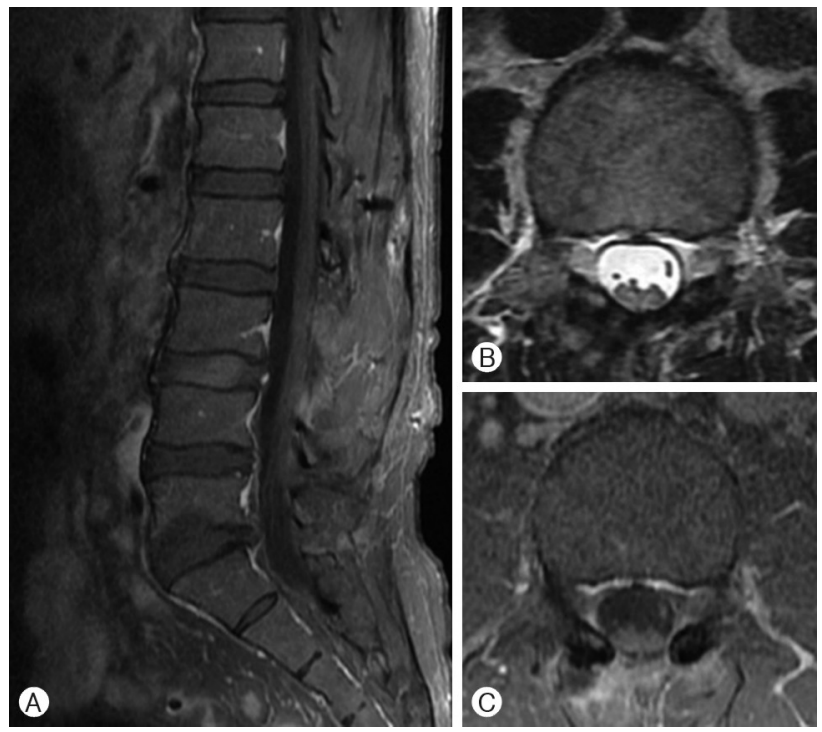

Fig. 4. Magnetic resonance image of 1 year from the surgery did not show any evidence of residual or recurrent tumor. The pain of the patient disappeared and he was also neurologically free.

associated tumors such as retinal or CNS HBL, renal cell carcinoma, pancreatic cysts, and neuroendocrine tumors. Genetic study of VHL gene could be supplemented to confirm the diagnosis. The patient had no family history of VHL syndrome; hence, possibility of VHL could be excluded.

The patient underwent laminectomy, and the tumor was exposed.
Upon dura incision, a $2.6-\mathrm{cm}$ hard and well-demarcated mass was observed (Fig. 2). A reddish and hypervascular tumor was located in the subpial layer of the filum terminale, and total en bloc resection of the mass was performed with a clear resection margin (Fig. 3). The operative field was carefully inspected, and tumor seeding and daughter nodules were not observed. Histological examination confirmed a benign stromal tumor consistent with spinal cord HBL (Fig. 3).

The patient's clinical symptoms improved markedly after surgery. MR images obtained on postoperative day 1 as well as 1 year after the surgery showed total resection of the tumor with no evidence of residual or recurrent tumors (Fig. 4). The spot-like lesion shown at preoperative MR imaging (MRI) around the venous engorgement was disappeared as well as solid tumor, so we could conclude that the spot lesion meant a part of venous engorgement rather than a daughter nodule.

The patient returned to our clinic complaining of pain in the right leg ten years after the surgery. An MR study of the lumbar spine showed an extraforaminal ruptured disc at L4-5 on the right side (Fig. 5), consistent with the patient's symptoms. In addition, four well-enhancing round nodules around the L3-4 level were noted (largest, $7.8 \mathrm{~mm}$; Fig. 5). The nodules were homogenously enhanced and located along the cauda equina. Even though the mass was not pathologically confirmed, the diagnosis of delayed recurrence of HBL could be made with characteristic findings of image study. The patient underwent L4-5 discectomy by a paraspinal approach because of persistent, radiating pain despite the root block. The pain immediately alleviated after surgery. The recurrent tumors did not evoke any neurological symptoms requiring treatment. Considering 

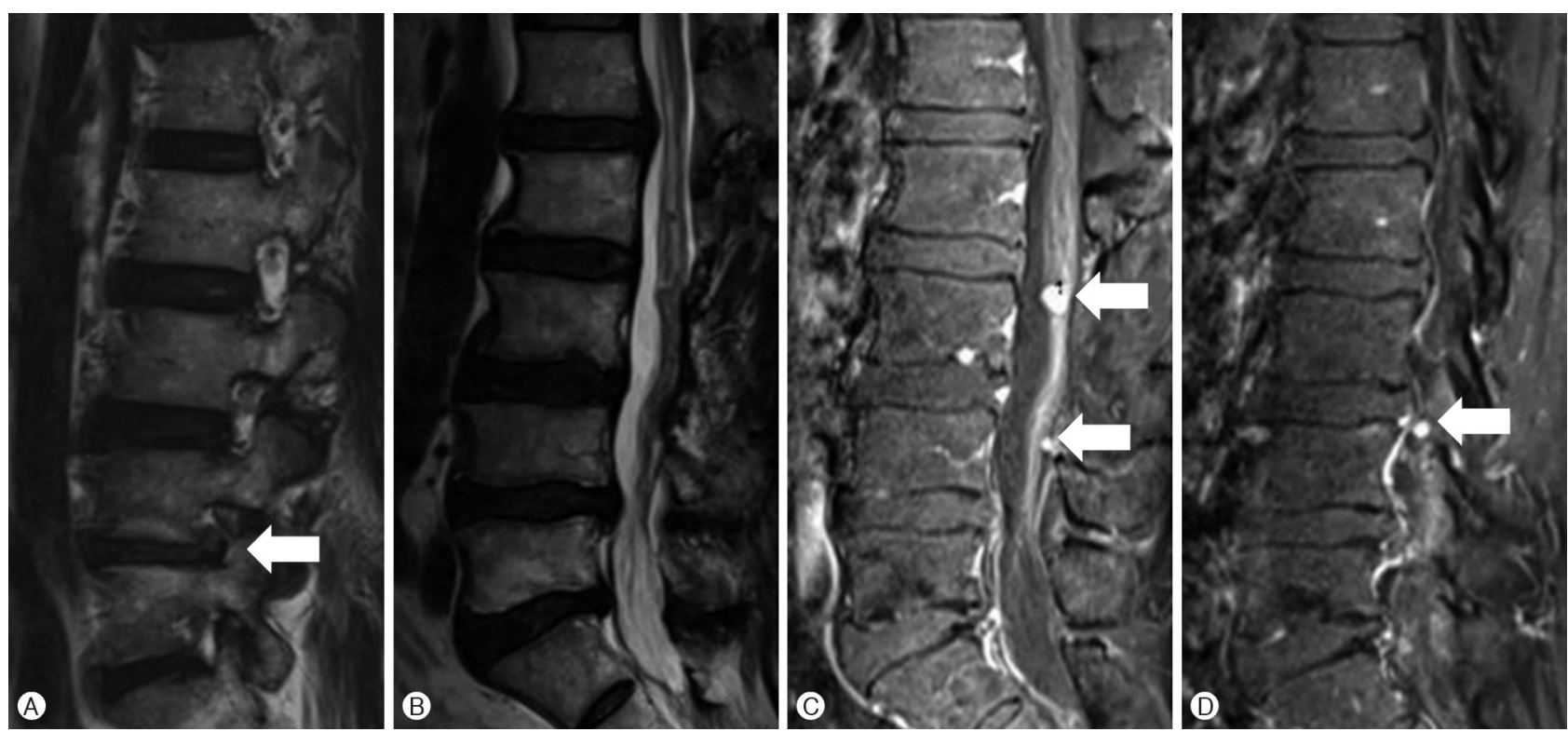

Fig. 5. Follow-up magnetic resonance imaging was performed 10 years after the tumor removal. The patient complained of a pain in the right leg. A ruptured disc at L4-5 was found on the right neural foramen (white thick arrow), which was thought to be the cause of the radia ting pain. The pain was dramatically but temporarily improved after a transforaminal root block. The 4 homogenous well contrast enhancing nodules (white arrows, largest: $7.8 \mathrm{~mm}$ ) are observed at L3, L4 level. The mass were along the spinal nerve roots, encroaching lateral recess. With considering the radiological characteristics, the nodules were consistent with the diagnosis of be the delayed recurrence of the hemangioblastoma.

the benign nature of spinal cord HBLs, observation of the tumors through repeated follow-up imaging studies was planned. If tumor growth is noted, surgical resection or adjunctive radiotherapy can be considered as treatment options.

\section{DISCUSSION}

Spinal HBL is a benign, highly vascularized tumor that can occur sporadically or in relation to $\mathrm{VHL}^{7}$. GTR is the treatment of choice for patients presenting with neurological symptoms. Preoperative embolization of the tumor may be considered to reduce intraoperative blood loss and shrink the tumor to facilitate surgical resection ${ }^{4)}$. Radiosurgery is an option for treatment in unresectable locations and remnant tumors after surgery".

VHL-related spinal HBL tends to develop multiple lesions and recurs frequently after surgery. In contrast, sporadic-type spinal HBL is a benign tumor, which is thought to rarely recur after $\mathrm{GTR}^{7,14)}$. In general, recurrence after surgery is associated with subtotal or incomplete resection ${ }^{18}$.

There have been previous reports of relapsed HBL after complete surgical resection, ${ }^{8,14,15}$. Lee et al. ${ }^{7}$ reported that microscopic remnants during surgical resection or the presence of tumor cells in the cystic wall could be related to recurrence in cases of recurrent cerebellar $\mathrm{HBL}$ without VHL. In posterior fossa HBLs with mural nodules, remnant cystic walls from a previous operation may regrow because of diffusion of cerebrospinal fluid. In addition, the presence of multiple satellite lesions that cannot be detected in the operative field can become a cause of recurrence ${ }^{3)}$. The HBLs are usually hypenascular, and excessive bleeding may interfere with complete surgical excision.

Efforts to minimize residual tumors can prevent or reduce tumor recurrence. According to Gläsker et al. ${ }^{2)}$, dissection of the tumor from the nerve sheath alone is insufficient in avoiding recurrence. Surgeons should try to identify and resect the fascicles of origin. Intraoperative fluorescence imaging, such as indocyanine green, could be useful in preventing subtotal resection of the tumor. Li et al. ${ }^{9}$ reported intraoperative fluorescence as helpful for effective GTR of spinal HBL, with their results showing no postoperative recurrence. Fluorescence angiography could help identify subtle lesions located in the intramedullary space ${ }^{9)}$.

Stereotactic radiosurgery (SRS) is also available for treating spinal HBLs. Gamma knife surgery or CyberKnife radiosurgery has been reported as a successful treatment option for remnant, inoperable, and multiple lesions in cranial and spinal HBLs. SRS for recurrent cranial HBLs has proven beneficial in both the sporadic and VHLrelated types ${ }^{5,11)}$. There have been a few studies on the effects of SRS in recurrent spinal HBL cases. In a study by Ryu et al. ${ }^{12}$, SRS was effective in three recurrent spinal HBL cases. For patients who are not candidates for surgery, SRS can be regarded as an effective and safe treatment option to control disease and improve survival and is an attractive alternative to multiple surgical procedures ${ }^{11)}$.

Although surgical resection is the treatment of choice for spinal HBL, radiotherapy also plays a role. Fractionated radiotherapy could be useful in eloquent areas of the brain, intradural spinal cord lesions, or multiple tumors with extensive lesions ${ }^{6}$. However, radiothe- 
rapy has limitations such as radiotoxicity. Dose-response relationships have to be closely monitored to prevent radiotoxicity. Moreover, in multiple lesions, the effective radiation dose differs according to the histopathologic characteristics of each tumor. Therefore, radiotherapy is considered in cases of VHL-related spinal HBLs rather than in sporadic types.

Complete surgical resection of the spinal HBL is the most effective treatment to prevent a recurrence. However, tumors can still recur after GTR even in sporadic cases. Shin et al. ${ }^{13}$ reported five recurrent sporadic spinal HBL tumors in 16 patients who had undergone prior surgery. Among the five patients with recurrent tumors, three patients had undergone total resection and the others had undergone subtotal resection. The average period of recurrence from surgery was 6.4 years and ranged from 1 to 13.1 years. Sun et al. ${ }^{14)}$ reported 14 cases of sporadic spinal HBL, and one patient showed a delayed recurrence 15 years affer GTR. Asymptomatic recurrence may be found incidentally in follow-up imaging studies, as in our case. Li et al. ${ }^{87}$ reported a case of tumor recurrence, noting that all recurrent HBLs presented with neurological symptoms and were surgically removed 4.5 years affer the initial surgery. Two months after the second surgery, another nodular recurrence was found during followup MRI. The patient did not complain of any neurological symptoms, and the recurrent tumor showed spontaneous growth arrest and remained stable for 9 years.

In our case, we achieved GTR of the tumor using a microscope without any evidence of residual mass in the operative field. Residual tumor masses were not observed tumor at one year after the surgery but incidentally multiple-well enhancing masses were found around the previous tumor lesion and along the cauda equina, ten years thereafter. The lesions showed vivid contrast enhancement which suggests that HBLs are recurred. HBLs usually arise as solitary expan- sile masses; however, in our case, the tumor had a multilobular appearance and intermingled with multiple lumbar roots, which made surgical removal significantly more complex. In total, this delayed recurrence might be related to the subtle microscopic remnant tumor or tumor seeding that could not be identified in the operative field slowly growing over the years. Therefore, meticulous observation of the origin and remnants or satellite lesions of the tumor is necessary, especially when surgeons resected multilobular mass, to decrease the possibility of recurrence.

According to the reported articles and our case review, delayed recurrent tumors were often discovered several years after surgery. Because of the low recurrence rate and slow growth, recurrence may be described as having a delayed pattern. Therefore, after total resection of HBLs, even in sporadic cases, follow-up imaging studies should be continued for a long time. In particular, if preoperative HBLs consist of high-risk factors for recurrences, such as VHL syndrome, cysts, and a multilobular shape, careful long-erm follow-up is necessary. Considering the reported cases, follow-up could be conducted for up to 10 to 15 years after the surgical excision.

The limitation of this study is that there were few long-term follow-up studies on spinal cord HBL surgery to suggest guidelines for the imaging study follow-up. More cases need to be supplemented. Also, the recurrence was found ten years after the surgery in our case. The only prior imaging study was performed one year after the surgery; due to the time gap of the imaging studies, we cannot specify the exact time of recurrence. In addition, since the patient did not show any neurologic symptoms related to recurrent lesions, we did not perform a second operation to confirm the pathologic diagnosis. The diagnosis of recurrence HBLs was made based on consistent radiological findings. We could not completely rule out newly developing VHL syndrome because we did not check VHL gene study. Longer follow-up and additional imaging studies are planned to surveil the tumor growth. We plan to conduct a genetic study to exclude VHL syndrome if the tumor grows as large as the need for surgery in the future.

\section{CONCLUSION}

Complete surgical excision is the treatment of choice for spinal cord HBL. Delayed recurrence may be observed even affer GTR in sporadic cases. Long-term follow-up should be performed, and resection surgery or radiosurgery can be considered in cases of symptomatic recurrence.

\section{CONFLICTS OF INTEREST}

No potential conflict of interest relevant to this article was reported.

\section{REFERENCES}

1. Bukhari SS, Bari ME, Ahmad Z, Din NU: Spinal cord hemangioblastomas with a focus on clinical presentation, diagnosis, and treatment at a tertiary care hospital of Karachi, Pakistan: A retrospective chart review. Surg Neurol Int 12:24, 2021

2. Gläsker S, Berlis A, Pagenstecher A, Vougioukas VI, Van Velthoven $\mathrm{V}$ : Characterization of hemangioblastomas of spinal nerves. Neurosurgery 56:503-509, 2005

3. Gupta S, Pal L, Sardhara JC, Jaiswal AK, Srivastava A, Mehrotra A, et al.: Recurrent or symptomatic residual posterior fossa hemangioblastomas: how are they different from their primary counterparts? Acta Neurochir (Wien) 159:1497-1510, 2017

4. Hussain I, Parker WE, Barzilai O, Bilsky MH: Surgical management of intramedullary spinal cord tumors. Neurosurg Clin N Am 31:237-249, 2020

5. Jawahar A, Kondziolka D, Garces YI, Flickinger JC, Pollock $\mathrm{BE}$, Lunsford LD: Stereotactic radiosurgery for hemangioblastomas of the brain. Acta Neurochir (Wien) 142:641-645, 2000

6. Koh ES, Nichol A, Millar BA, Ménard C, Pond G, Laperriere NJ: Role of fractionated external beam radiotherapy in hemangioblastoma of the central nervous system. Int J Radiat Oncol Biol Phys 69:1521-1526, 2007

7. Lee GJ, Jung TY, Kim IY, Jung S, Jang WY, Moon KS, et al.: The clinical experience of recurrent central nervous system hemangioblastomas. Clin Neurol Neurosurg 123:90-95, 2014

8. Li AY, Post AF, Dai JB, Choudhri TF: Spontaneous arrest of sporadic spinal hemangioblastoma growth after postoperative nodular recurrence: Case report. Cureus 10:e3380, 2018

9. Li X, Wang J, Niu J, Hong J, Feng Y: Diagnosis and micro- 
surgical treatment of spinal hemangioblastoma. Neurol Sci 37: 899-906, 2016

10. Lonser RR, Butman JA, Huntoon K, Asthagiri AR, Wu T, Bakhtian $\mathrm{KD}$, et al.: Prospective natural history study of central nervous system hemangioblastomas in von Hippel-Lindau disease. J Neurosurg 120:1055-1062, 2014

11. Moss JM, Choi CY, Adler JR, Jr., Soltys SG, Gibbs IC, Chang SD: Stereotactic radiosurgical treatment of cranial and spinal hemangioblastomas. Neurosurgery 65:79-85, 2009

12. Ryu SI, Kim DH, Chang SD: Stereotactic radiosurgery for hemangiomas and ependymomas of the spinal cord. Neurosurg Focus 15:E10, 2003

13. Shin DA, Kim SH, Kim KN, Shin HC, Yoon DH: Surgical management of spinal cord haemangioblastoma. Acta Neurochir (Wien) 150:215-220, 2008

14. Sun H, Özduman K, Usseli M, Özgen S, Pamir MN: Sporadic spinal hemangioblastomas can be effectively treated by microsurgery alone. World Neurosurg 82:836-847, 2014

15. Takai K, Taniguchi M, Takahashi H, Usui M, Saito N: Comparative analysis of spinal hemangioblastomas in sporadic disease and Von Hippel-Lindau syndrome. Neurol Med Chir (Tokyo) 50:560-567, 2010

16. Varshney N, Kebede AA, Owusu-Dapaah H, Lather J, Kaushik M, Bhullar JS: A review of Von Hippel-Lindau syndrome. J Kidney Cancer VHL 4:20-29, 2017

17. Wang H, Zhang L, Wang H, Nan Y, Ma Q: Spinal hemangioblastoma: surgical procedures, outcomes and review of the literature. Acta Neurol Belg 121:973-981, 2021

18. Yousef A, Rutkowski MJ, Yalcin CE, Eren OC, Caliskan I, Tihan T: Sporadic and Von-Hippel Lindau disease-associated spinal hemangioblastomas: institutional experience on their similarities and differences. J Neurooncol 143:547-552, 2019 